\begin{tabular}{ll}
\hline Frontiers in Heat and Mass Transfer & $\begin{array}{l}\text { Frontiers } \\
\text { ind } \\
\text { Mass trand }\end{array}$ \\
\hline
\end{tabular}

\title{
EFFECTS OF NON-UNIFORM SLOT SUCTION/INJECTION AND CHEMICAL REACTION ON MIXED CONVECTIVE MHD FLOW ALONG A VERTICAL WEDGE EMBEDDED IN A POROUS MEDIUM
}

\author{
M. Ganapathirao ${ }^{\mathrm{a}, *}$, Ali J. Chamkha ${ }^{\mathrm{b}}$, N. Srinivasa Rao ${ }^{\mathrm{c}}$ \\ ${ }^{a}$ Mathematics \& Basic Sciences, NIIT University, Neemrana, NH-8, Delhi-Jaipur Highway, Alwar District, Rajasthan, 301705, India \\ ${ }^{\mathrm{b}}$ Mechanical Engineering Department, Prince Sultan Endowment for Energy and Environment, Prince Mohammad Bin Fahd University, Al-Khobar \\ 31952, Saudi Arabia \\ ${ }^{\mathrm{c}}$ Division of Mathematics, Department of Science and Humanities, Vignan's Foundation for Science, Technology and Research, Vadlamudi, Guntur, \\ Andhra Pradesh, 522213, India
}

\begin{abstract}
In this investigation, our objective is to study the effect of non-uniform slot suction or injection into a steady mixed convective MHD boundary layer flow over a vertical wedge embedded in a porous medium in the presence of chemical reaction. The wall of the wedge is embedded in a uniform porous medium in order to allow possible fluid wall suction or injection. The surface of the wedge is maintained at a variable wall temperature and concentration. The fluid is assumed to be viscous, incompressible and electrically conducting; and the magnetic field is applied transversally in the direction of the flow. The governing boundary layer equations are transformed into a set of non-similar and non-dimensional equations by using suitable coordinate transformations. Non-similar solutions are obtained numerically by solving coupled non-linear partial differential equations using an implicit finite difference scheme in combination with the quasi-linearization technique. Comparisons with previously published works are performed and excellent agreement between the results is obtained. A parametric study of the physical parameters is conducted and a representative set of numerical results for the velocity, temperature and concentration distributions, as well as the local skin friction coefficient and the local Nusselt and Sherwood numbers are illustrated graphically to show interesting features of the solutions.
\end{abstract}

Keywords: non-similar solution; non-uniform slot suction; chemical reaction; porous medium; variable wall temperature.

\section{INTRODUCTION}

The study and analysis of heat and mass transfer in porous media has been the subject of many investigations due to their frequent occurrence in industrial and technological applications. Examples of some applications are geothermal reservoirs, drying of porous solids, thermal insulation, enhanced oil recovery and many others. There has been a renewed interest in studying magnetohydrodynamic(MHD) flow and heat and mass transfer aspects in various geometries due to the effect of magnetic field on the flow control and on the performance of many systems using electrically conducting fluids such as liquid metals, water mixed with little acid and others. In recent years, MHD flow problems have become important in industry, since many metallurgical processes involve the cooling of continuous strips or filaments. By drawing them in an electrically conducting fluid in the presence of a magnetic field, the rate of cooling can be controlled. The reason for studying the effect of the magnetic field on the flow through porous media is that the fluids are electrically conducting in geothermal regions and hence, these can be significantly influenced by the magnetic field. Magnetic field effects are encountered in different technological applications such as purification of molten metals, nuclear reactor coolers, metal casting, geothermal energy extraction and many others (Selimefendigil and Öztop, 2018, 2019a, 2019b).

Many practical diffusive operations involve the molecular diffusion of a species in the presence of chemical reaction within or at the boundary. Chemical reaction can be modeled as either heterogeneous or homogeneous processes which depends on whether it occurs at an interface or as a single-phase volume reaction. A homogeneous reaction is one that occurs uniformly throughout a given phase. The species generation in a homogeneous reaction is analogous to internal source of heat generation. On the other hand, a heterogeneous reaction takes place in a restricted region or within the boundary of a phase. A few representative fields of interest in which combined heat and mass transfer play an important role in the design of chemical processing equipment, formation and dispersion of fog, distribution of temperature, moisture over agricul-

${ }^{*}$ Corresponding author. Email: ganapathirao.m@gmail.com 
tural fields, groves of fruit trees, damage of crops due to freezing, food processing and cooling towers. In most of cases of chemical reaction, the reaction rate depends on the concentration of the species itself. One of the simplest chemical reactions is the first order reaction in which the rate of reaction is directly proportional to the species concentration. For example, formation of smog is a first-order homogeneous chemical reaction. Chemical reaction effect on heat and mass transfer laminar boundary layer flow have been discussed by many authors (Devi and Kandasamy, 2002; Kandasamy et al., 2005, 2008, 2010) in various situations. Effect of chemical reaction in the presence of uniform magnetic field plays an important role on the concentration field. The flow of a fluid past a wedge is of fundamental importance since this type of flow constitutes a general and wide class of flows in which the free stream velocity is proportional to the power of the length coordinate measured from the apex. In particular, the problem concerned with MHD flow and chemical reaction, heat and mass transfer over a vertical wedge with suction or injection may find applications in polymer technology, metallurgy and dyeing industries.

The study of boundary layer flow over a vertical wedge with suction or injection has attracted considerable attention of researchers due to frequent occurrence in many branches of science and technology. The process of suction and injection has an importance in many engineering applications such as in the design of thrust bearing and radial diffusers, thermal oil recovery, boundary layer control, skin friction reduction on high speed aircraft and flow separation. For example, suction is used in chemical processes to remove reactants and injection is used to add reactants, cool the surface, prevent corrosion or scaling, and reduce the drag. Fluid injection is widely used technique in the aeronautical industry. It is used, for example, for cooling gas turbine blades and for controlling transition and/or separation of the boundary layer over airplane control surfaces. Therefore, it is of great practical significance for the aerodynamicist and the thermal analyst to predict the effect of injection on the flow pattern and temperature distribution around a turbine blade or any other piece of machinery.

In many investigations, notable contribution on convection flows over a wedge was made by Watanabe (1990) who studied the thermal boundary layer over a wedge with uniform suction or injection in forced flow. Later, Watanabe et al. (1994) investigated the theoretical analysis of mixed convection flow over a wedge with uniform suction or injection. A uniform suction or injection effect on wedge flow including heat flux was studied by Yih (1998a). Further, the effect of transverse magnetic field on the flow over a wedge with uniform suction or injection was studied by Kafoussias and Nanousis (1997) and Nanousis (1999). Ishak et al. (2007) investigated the flow past a moving wedge with suction or injection. Kumari and Gorla (1997) discussed the combined convection along a non-isothermal wedge in porous medium. Later, Kumari et al. (2001) have investigated the mixed convection flow over a vertical wedge embedded in a highly porous medium in the presence of uniform suction or injection. MHD flow adjacent to a non-isothermal wedge in forced convection was studied by Yih (1999). Hydromagnetic free convection flow over a vertical cone and a wedge in porous medium was carried out by Chamkha (1996) and Chamkha et al. (2000). Gorla et al. (2012) analyzed the combined convective heat transfer in non-Newtonian boundary layer flow over a wedge in porous medium. Mixed convection flow over a vertical plate with localized heating (cooling), magnetic field and suction or injection is investigated by Chamkha et al. (2004). Moreover, various studies (Kandasamy et al., 2008, 2010; Muhaimin and Khamis, 2010) showed MHD flow along a wedge in porous medium with uniform suction or injection. The effect of variable wall temperature and concentration on the flow over a wedge in porous media was studied by Koh and Hartnett (1961) and Yih (1998b). Devi and Kandasamy (2002) and Kandasamy et al. (2005) studied the chemical reaction effect on mixed convective flow along a wedge with suction or injection. Almost all aforementioned studies were primarily concerned with uniform suction or injection (i.e. mass suction or injection occurs in an entire porous section of the body surface).

In many cases, mass suction or injection from a wall slot (i.e. mass suction or injection occurs in a small porous section of the body surface, while there is no suction or injection in the remaining part of the body surface) into the boundary layer is of interest for various eventual applications including thermal protection, fuel injection in ramjet engines, energizing of the inner portion of boundary layers in adverse pressure gradients and skin friction reduction on high speed aircraft. In fact, mass suction or injection through slot strongly influences the development of a boundary layer along a surface and in particular can prevent or at least delay the separation of the viscous region. Uniform slot injection into a laminar boundary layer by taking the interaction between the boundary layer and oncoming stream have been discussed by Smith and Stewartson (1973), Napolitano and Messick (1980) and Riley (1976). Smith and Stewartson (1973), and Napolitano and Messick (1980), considered uniform normal injection into a boundary layer in supersonic flow and subsonic flow respectively; and Riley (1976) who considers uniform oblique injection into a boundary layer in a supersonic flow. For the case of uniform normal injection, there is a finite discontinuity in the pressure gradient at each of the leading and trailing edges of the slot. For the case of uniform oblique injection. Riley showed that at the edges of the slot, the pressure gradient is infinite and, the shear stress is also discontinuous and unbounded.

Uniform suction or injection in a slot causes finite discontinuities at the leading and trailing edges of the slot which can be avoided by choosing a non-uniform suction or injection in a slot as discussed by Minkowycz et al. (1988) and in an early work by Riley (1981) who considered non-uniform slot injection into a laminar boundary layer in both supersonic and subsonic flow. Recently, a few studies (Roy and Saikrishnan, 2003, 2004; Saikrishnan and Roy, 2003; Datta et al., 2006; Ravindran and Ganapathirao, 2013; Ganapathirao et al., 2014; Samyuktha et al., 2016) reported non-uniform slot suction or injection into boundary layer flow past yawed cylinder, sphere, cone, rotating sphere, cylinder and plate.

The consequence of avoiding finite discontinuities at the leading and trailing edges of the slot helps to obtain smooth solutions for a large value of the mass transfer parameter without the difficulties of numerical instability. It may be noted that such difficulties are pointed out by previous researchers for uniform mass transfer studies with finite discontinuities at the leading and trailing edges of the slot which has been avoided in the present study. Thus, the present study differs from the studies by Smith and Stewartson (1973), Napolitano and Messick (1980) and Riley (1976) which had finite discontinuities.

In the current study, laminar mixed convective MHD flow over a vertical wedge in porous media with slot suction/injection has been investigated under the influence of first-order chemical reaction. The problem dealing with mixed convection flow past a wedge with suction or injection is of interest in relation to boundary layer control. The lower energy fluid near the wall is removed from the boundary layer through non-uniform slot suction and this helps to control the back flow for negative values of buoyancy parameter. On the other hand, non-uniform slot injection helps to reduce the skin friction, heat and mass transfer coefficients at a particular stream-wise location on the surface. The potential application of non-uniform slot suction/injection is widely used in the aircraft for reducing heat transfer across turbine blades and controlling transition and /or separation of boundary layers over airplane control surfaces. Numerical simulations have been performed to analyze the impact of various pertinent parameters on the convective heat and mass transfer characteristics. The results of this study may be used for a wide range of thermal engineering applications such as geothermal systems, crude oil extractions, ground water pollution, thermal insulation, solid matrix heat exchangers, storage of nuclear wastes, etc. 


\section{MATHEMATICAL FORMULATION}

Consider a two-dimensional, steady mixed convective laminar boundary layer flow of an electrically conducting fluid over a vertical wedge with half angle $\frac{\pi \gamma}{2}$ immersed in a highly porous medium. It is assumed that the flow moves parallel to the surface of the wedge in the upward direction with free stream velocity $u_{\infty}$ and the gravitational acceleration $g$ acts downward parallel to the axis of the wedge. Both the wall temperature $T_{w}$ and concentration $C_{w}$ are assumed to vary with distance from the leading edge along the wall according to a power law model. Free stream temperature $T_{\infty}$ and concentration $C_{\infty}$ are taken as constants, where $T_{w}>T_{\infty}$ corresponds to a heated wedge and $T_{w}<T_{\infty}$ corresponds to a cooled wedge. The coordinate $x$ is measured along the surface of the wedge from the apex and the coordinate $y$ is measured normal to it. The physical model and coordinate system is shown in Fig. 1.

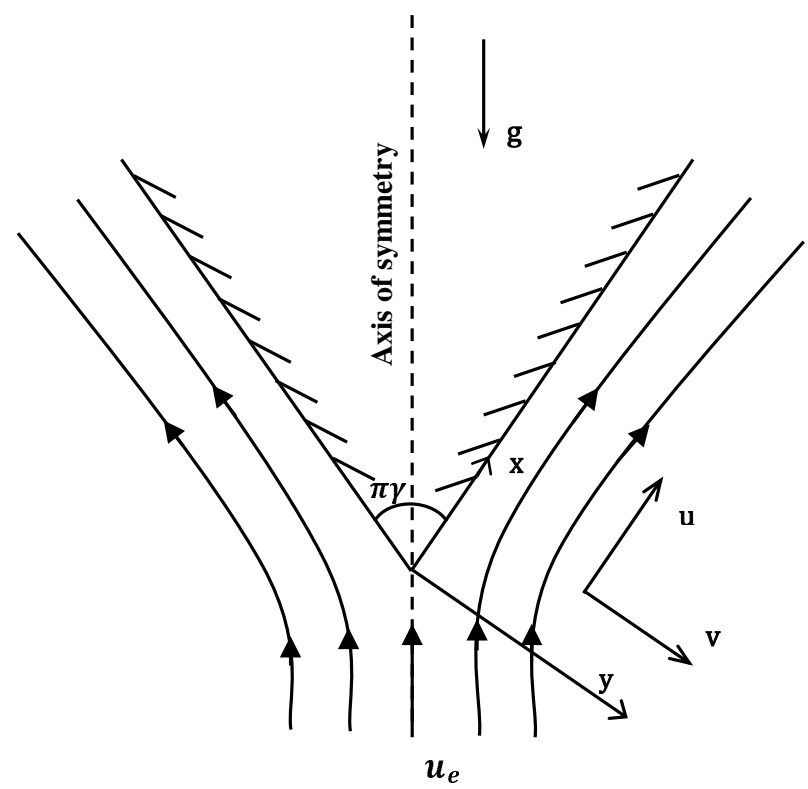

Fig. 1 Physical model and coordinate system.

A uniform magnetic field of strength $B$ is applied in the $y$ - direction, and a chemical reaction takes place in the flow. The magnetic Reynolds number is assumed to be small so that the induced magnetic field can be neglected in comparison to the applied magnetic field. In addition to this, there is no applied electric field and both the Hall effect, viscous dissipation and Joule heating are neglected. Here, the porous medium is considered to be isotropic and homogeneous. The porous medium causes the flow resistance which is taken to be proportional to the velocity. Non-uniform slot suction/injection is imposed at the wedge surface. The fluid is assumed to be Newtonian, electrically conducting and has constant properties except the density in the buoyancy force term of the momentum equation.

Under the above assumptions along with Boussinesq approximation, the boundary layer equations governing the non-similar flow over a wedge embedded in a highly porous medium can be expressed as (Schlichting and Gersten, 2000):

$$
\begin{gathered}
\frac{\partial u}{\partial x}+\frac{\partial v}{\partial y}=0 \\
u \frac{\partial u}{\partial x}+v \frac{\partial u}{\partial y}=u_{e} \frac{\partial u_{e}}{\partial x}+\nu \frac{\partial^{2} u}{\partial y^{2}} \\
+\left[g \beta\left(T-T_{\infty}\right)+g \beta^{*}\left(C-C_{\infty}\right)\right] \cos \left(\frac{\pi \gamma}{2}\right)
\end{gathered}
$$

$$
\begin{gathered}
-\frac{\sigma B^{2}}{\rho}\left(u-u_{e}\right)-\frac{g}{K_{1}}\left(u-u_{e}\right) \cos \left(\frac{\pi \gamma}{2}\right), \\
u \frac{\partial T}{\partial x}+v \frac{\partial T}{\partial y}=\frac{\nu}{\operatorname{Pr}} \frac{\partial^{2} T}{\partial y^{2}}, \\
u \frac{\partial C}{\partial x}+v \frac{\partial C}{\partial y}=\frac{\nu}{S c} \frac{\partial^{2} C}{\partial y^{2}}-k_{c}\left(C-C_{\infty}\right) .
\end{gathered}
$$

The physical boundary conditions of the problem are

$$
\begin{aligned}
& u=0, \quad v=v_{w}, \quad T=T_{w}(\bar{x})=T_{\infty}+b_{1}(\bar{x})^{n}, \\
& C=C_{w}(\bar{x})=C_{\infty}+b_{2}(\bar{x})^{n} \text { at } y=0 \\
& u \rightarrow u_{e}, \quad T \rightarrow T_{\infty}, \quad C \rightarrow C_{\infty} \text { as } y \rightarrow \infty
\end{aligned}
$$

where $v_{w}$ is the suction or injection velocity, $u_{e}$ is the velocity at the edge of the boundary layer and $n$ is the wall temperature/concentration exponent.

Applying the following transformations:

$$
\begin{gathered}
\eta=y\left(\frac{m+1}{2} \frac{u_{e}}{x \nu}\right)^{1 / 2}, \quad \psi(x, y)=\left(\frac{2}{m+1} x \nu u_{e}\right)^{1 / 2} f(\bar{x}, \eta) \\
u_{e}=u_{\infty}(\bar{x})^{m}, \quad \bar{x}=\frac{x}{L}, \quad m=\frac{\bar{x}}{u_{e}} \frac{d u_{e}}{d \bar{x}}=\frac{\gamma}{2-\gamma} \\
G(\bar{x}, \eta)=\frac{T-T_{\infty}}{T_{w}-T_{\infty}}, \quad H(\bar{x}, \eta)=\frac{C-C_{\infty}}{C_{w}-C_{\infty}} \\
u=\frac{\partial \psi}{\partial y}, \quad v=-\frac{\partial \psi}{\partial x} \\
u=u_{e} F(\bar{x}, \eta), \quad f_{\eta}(\bar{x}, \eta)=F(\bar{x}, \eta) \\
v=-\left(\frac{2}{m+1} \frac{\nu u_{e}}{x}\right)^{1 / 2} \times \frac{1}{2}\left[(m+1) f+2 \bar{x} f_{\bar{x}}+(m-1) \eta F\right]
\end{gathered}
$$

It is found that Eq. (1) is trivially satisfied, and Eqs. (2) - (4) reduce to non-dimensional form as follows:

$$
\begin{aligned}
& F_{\eta \eta}+f F_{\eta}+\frac{2 m}{m+1}\left(1-F^{2}\right)+\frac{2}{m+1} \lambda N_{1}[G+S H] \cos \left(\frac{\pi \gamma}{2}\right) \\
& +\frac{2}{m+1} N_{2}\left[M+K \cos \left(\frac{\pi \gamma}{2}\right)\right](1-F) \\
& =\frac{2}{m+1} \bar{x}\left(F F_{\bar{x}}-F_{\eta} f_{\bar{x}}\right) \text {, } \\
& P r^{-1} G_{\eta \eta}+f G_{\eta}-\frac{2 n}{m+1} F G=\frac{2}{m+1} \bar{x}\left(F G_{\bar{x}}-G_{\eta} f_{\bar{x}}\right), \\
& S c^{-1} H_{\eta \eta}+f H_{\eta}-\frac{2 n}{m+1} F H-\frac{2}{m+1} S c \Delta N_{2} H \\
& =\frac{2}{m+1} \bar{x}\left(F H_{\bar{x}}-H_{\eta} f_{\bar{x}}\right), \\
& \text { where } N_{1}=\bar{x}\left(\frac{u_{\infty}}{u_{e}}\right)^{2}, N_{2}=\bar{x}\left(\frac{u_{\infty}}{u_{e}}\right) \text {, } \\
& \lambda=\frac{G r_{L}}{R e_{L}^{2}}, \quad \lambda^{*}=\frac{G r_{L}^{*}}{R e_{L}^{2}}, \quad S=\frac{\lambda^{*}}{\lambda}, R e_{L}=\frac{u_{\infty} L}{\nu}, \\
& G r_{L}=\frac{g \beta L^{3}\left(T_{w}-T_{\infty}\right)}{\nu^{2}}, G r_{L}^{*}=\frac{g \beta^{*} L^{3}\left(C_{w}-C_{\infty}\right)}{\nu^{2}}, \\
& K=\frac{K_{2}}{R e_{L}}, \quad K_{2}=\frac{g L^{2}}{\nu K_{1}}, \quad M=\frac{H a^{2}}{R e_{L}}, H a^{2}=\frac{\sigma B^{2} L^{2}}{\mu}, \\
& \operatorname{Pr}=\frac{\nu}{\alpha}, \quad S c=\frac{\nu}{D}, \Delta=\frac{k_{c} L}{u_{\infty}}, R e_{x}=\frac{u_{e} x}{\nu} .
\end{aligned}
$$

The associated boundary conditions become

$$
\begin{array}{r}
f=f_{w}, \quad F=0, \quad G=1, \quad H=1 \quad \text { at } \eta=0 \\
F \rightarrow 1, \quad G \rightarrow 0, \quad H \rightarrow 0 \quad \text { as } \eta \rightarrow \infty
\end{array}
$$




$$
\begin{gathered}
\text { where } f=\int_{0}^{\eta} F d \eta+f_{w}, f_{w} \text { is given by } \\
\left(\frac{m+1}{2}\right) f_{w}+\bar{x}\left(f_{\bar{x}}\right)_{w}=-\frac{v_{w}}{u_{\infty}}\left[\left(\frac{m+1}{2}\right) R e_{L}\right]^{1 / 2} \times(\bar{x})^{\frac{1-m}{2}}
\end{gathered}
$$

If we put $\xi=(\bar{x})^{(1-m) / 2}$, then Eqs. (7) - (9) reduce to:

$$
\begin{gathered}
F_{\eta \eta}+f F_{\eta}+\frac{2 m}{m+1}\left(1-F^{2}\right)+\frac{2}{m+1} \lambda N_{3}[G+S H] \cos \left(\frac{\pi \gamma}{2}\right) \\
+\frac{2}{m+1} N_{4}\left[M+K \cos \left(\frac{\pi \gamma}{2}\right)\right](1-F) \\
=\left(\frac{1-m}{1+m}\right) \xi\left(F F_{\xi}-F_{\eta} f_{\xi}\right), \\
P r^{-1} G_{\eta \eta}+f G_{\eta}-\frac{2 n}{m+1} F G=\left(\frac{1-m}{1+m}\right) \xi\left(F G_{\xi}-G_{\eta} f_{\xi}\right), \\
S c^{-1} H_{\eta \eta}+f H_{\eta}-\frac{2 n}{m+1} F H-\frac{2}{m+1} S c \Delta N_{4} H \\
=\left(\frac{1-m}{1+m}\right) \xi\left(F H_{\xi}-H_{\eta} f_{\xi}\right),
\end{gathered}
$$

where

$$
N_{3}=\xi^{\frac{2(1-2 m)}{1-m}}, \quad N_{4}=\xi^{2} .
$$

The corresponding boundary conditions become

$$
\begin{array}{r}
f=f_{w}, \quad F=0, \quad G=1, \quad H=1 \text { at } \eta=0 \\
F \rightarrow 1, \quad G \rightarrow 0, \quad H \rightarrow 0 \quad \text { as } \eta \rightarrow \infty
\end{array}
$$

and Eq. (11) becomes

$$
\left(\frac{m+1}{2}\right) f_{w}+\left(\frac{1-m}{2}\right) \xi\left(f_{\xi}\right)_{w}=-\frac{v_{w}}{u_{\infty}} \xi\left[\left(\frac{m+1}{2}\right) R e_{L}\right]^{1 / 2}
$$

On solving (16), we get

$f_{w}=-\frac{1}{u_{\infty}}\left(\frac{2}{1-m}\right)\left(\frac{m+1}{2}\right)^{1 / 2}\left(R e_{L}\right)^{1 / 2} \times \xi^{-\left(\frac{1+m}{1-m}\right)} \int_{0}^{\xi} \xi^{\frac{1+m}{1-m}} v_{w} \mathrm{~d} \xi$,

here $v_{w}$ is the surface mass transfer velocity with $v_{w}<0$ for suction and $v_{w}>0$ for injection.

A recent demand on the effect of surface mass transfer (suction or injection) through a wall slot for the present day aerodynamic problems, we have imposed slot suction or injection at the wedge surface. In fact, if we choose uniform suction or injection in a slot causes finite discontinuities at the leading and the trailing edges of the slot. Those discontinuities can be avoided by choosing non-uniform mass transfer in a slot and it was pointed out by many previous researchers (Riley, 1981; Roy and Saikrishnan, 2003, 2004; Saikrishnan and Roy, 2003; Datta et al., 2006).

Therefore, the consequence of avoiding finite discontinuities at the leading and trailing edges of the slot in the case of uniform mass transfer, we consider here an appropriate non-uniform slot mass transfer velocity $v_{w}$ as a sinusoidal function and is given by

$$
v_{w}=\left\{\begin{array}{lc}
-u_{\infty}\left(\frac{1-m}{2}\right)\left(\frac{2}{m+1}\right)^{1 / 2} & \left(R e_{L}\right)^{-1 / 2} A \xi^{-\left(\frac{1+m}{1-m}\right)} \times \\
\omega^{*} \sin \left\{\omega^{*}\left(\xi-\xi_{0}\right)\right\}, & \xi_{0} \leq \xi \leq \xi_{0}^{*} \\
0, & \text { otherwise }
\end{array}\right.
$$

Thus, the value of $f_{w}$ is obtained as:

$$
f_{w}= \begin{cases}0, & \xi \leq \xi_{0} \\ A \xi^{-\left(\frac{1+m}{1-m}\right)}\left[1-\cos \left\{\omega^{*}\left(\xi-\xi_{0}\right)\right\}\right], & \xi_{0} \leq \xi \leq \xi_{0}^{*} \\ A \xi^{-\left(\frac{1+m}{1-m}\right)}\left[1-\cos \left\{\omega^{*}\left(\xi_{0}^{*}-\xi_{0}\right)\right\}\right], & \xi \geq \xi_{0}^{*},\end{cases}
$$

here $\omega^{*}, \xi_{0}$ are the two free parameters which determine the slot length and slot location respectively. The function $v_{w}$ is continuous for all the values of $\xi$ and it has a non-zero value only in the interval $\left[\xi_{0}, \xi_{0}^{*}\right]$. The reason for taking this type of function is that it allows mass transfer to change slowly in the neighborhood of the leading and trailing edges of the slot. Thus, the mass transfer function is chosen in such a way that there is no discontinuity at the leading and trailing edges of the slot. The surface mass transfer parameter $A>0$ or $A<0$ indicates the suction or injection, respectively.

For practical applications, the major physical quantities of interest include the local skin friction coefficient

$$
C_{f x}=\frac{2 \mu\left(\frac{\partial u}{\partial y}\right)_{y=0}}{\rho u_{e}^{2}}=2\left(R e_{x}\right)^{-1 / 2}\left(\frac{m+1}{2}\right)^{1 / 2}\left(F_{\eta}\right)_{w}
$$

the heat transfer coefficient in terms of the local Nusselt number

$$
N u_{x}=\frac{x\left(-\frac{\partial T}{\partial y}\right)_{y=0}}{T_{w}-T_{\infty}}=-\left(R e_{x}\right)^{1 / 2}\left(\frac{m+1}{2}\right)^{1 / 2}\left(G_{\eta}\right)_{w}
$$

and the mass transfer coefficient in terms of the local Sherwood number

$$
S h_{x}=\frac{x\left(-\frac{\partial C}{\partial y}\right)_{y=0}}{C_{w}-C_{\infty}}=-\left(R e_{x}\right)^{1 / 2}\left(\frac{m+1}{2}\right)^{1 / 2}\left(H_{\eta}\right)_{w} .
$$

Thus,

$$
\begin{gathered}
C_{f x}\left(R e_{x}\right)^{1 / 2}=2\left(\frac{m+1}{2}\right)^{1 / 2}\left(F_{\eta}\right)_{w}, \\
N u_{x}\left(R e_{x}\right)^{-1 / 2}=-\left(\frac{m+1}{2}\right)^{1 / 2}\left(G_{\eta}\right)_{w}, \\
S h_{x}\left(R e_{x}\right)^{-1 / 2}=-\left(\frac{m+1}{2}\right)^{1 / 2}\left(H_{\eta}\right)_{w} .
\end{gathered}
$$

\section{METHOD OF SOLUTION}

The set of coupled non-linear partial differential equations (12) - (14) along with the boundary conditions (15) have been solved numerically by using an implicit finite difference scheme in combination with the quasi-linearization technique (Bellman and Kalaba, 1965; Inouye and Tate, 1974).

With the help of quasi-linearization technique, the coupled non-linear partial differential equations (12) - (14) are linearized and the linearized partial differential equations are given by

$$
\begin{gathered}
F_{\eta \eta}^{i+1}+X_{1}^{i} F_{\eta}^{i+1}+X_{2}^{i} F^{i+1}+X_{3}^{i} F_{\xi}^{i+1}+X_{4}^{i} G^{i+1}+X_{5}^{i} H^{i+1}=X_{6}^{i}, \\
G_{\eta \eta}^{i+1}+Y_{1}^{i} G_{\eta}^{i+1}+Y_{2}^{i} G_{\xi}^{i+1}+Y_{3}^{i} G^{i+1}+Y_{4}^{i} F^{i+1}=Y_{5}^{i} \\
H_{\eta \eta}^{i+1}+Z_{1}^{i} H_{\eta}^{i+1}+Z_{2}^{i} H_{\xi}^{i+1}+Z_{3}^{i} H^{i+1}+Z_{4}^{i} F^{i+1}=Z_{5}^{i} .
\end{gathered}
$$

The coefficient functions with iterative index $(i)$ are known and the functions with iterative index $(i+1)$ are to be determined.

The corresponding boundary conditions are:

$$
\begin{array}{r}
F^{i+1}=0, \quad G^{i+1}=1, \quad H^{i+1}=1 \quad \text { at } \eta=0 \\
F^{i+1} \rightarrow 1, \quad G^{i+1} \rightarrow 0, \quad H^{i+1} \rightarrow 0 \quad \text { as } \eta \rightarrow \infty
\end{array}
$$


The coefficients in Eqs. (21) - (23) are given by

$$
\begin{aligned}
& X_{1}^{i}=f+\left(\frac{1-m}{1+m}\right) \xi f_{\xi}, \\
& X_{2}^{i}=-\left(\frac{4 m}{m+1}\right) F-\left(\frac{1-m}{1+m}\right) \xi F_{\xi} \\
& -\frac{2}{m+1} N_{4}[M+K \cos (\pi \beta / 2)] \text {, } \\
& X_{3}^{i}=-\left(\frac{1-m}{1+m}\right) \xi F, \\
& X_{4}^{i}=\frac{2}{m+1} \lambda N_{3} \cos (\pi \beta / 2), \\
& X_{5}^{i}=\frac{2}{m+1} \lambda S N_{3} \cos (\pi \beta / 2), \\
& X_{6}^{i}=-\left(\frac{2 m}{m+1}\right)\left(1+F^{2}\right)-\left(\frac{1-m}{1+m}\right) \xi F F_{\xi} \\
& -\frac{2}{m+1} N_{4}[M+K \cos (\pi \beta / 2)] \text {; } \\
& Y_{1}^{i}=\operatorname{Pr}\left[f+\left(\frac{1-m}{1+m}\right) \xi f_{\xi}\right] \text {, } \\
& Y_{2}^{i}=-\operatorname{Pr}\left(\frac{1-m}{1+m}\right) \xi F, \\
& Y_{3}^{i}=-\operatorname{Pr}\left(\frac{2 n}{m+1}\right) F, \\
& Y_{4}^{i}=-\operatorname{Pr}\left(\frac{1-m}{1+m}\right) \xi G_{\xi}-\operatorname{Pr}\left(\frac{2 n}{m+1}\right) G, \\
& Y_{5}^{i}=-\operatorname{Pr}\left(\frac{1-m}{1+m}\right) \xi G_{\xi} F-\operatorname{Pr}\left(\frac{2 n}{m+1}\right) G F \text {; } \\
& Z_{1}^{i}=S c\left[f+\left(\frac{1-m}{1+m}\right) \xi f_{\xi}\right] \text {, } \\
& Z_{2}^{i}=-S c\left(\frac{1-m}{1+m}\right) \xi F, \\
& Z_{3}^{i}=-S c\left(\frac{2 n}{m+1}\right) F-S c\left(\frac{2}{m+1}\right) \Delta N_{4}, \\
& Z_{4}^{i}=-S c\left(\frac{1-m}{1+m}\right) \xi H_{\xi}-S c\left(\frac{2 n}{m+1}\right) H, \\
& Z_{5}^{i}=-S c\left(\frac{1-m}{1+m}\right) \xi H_{\xi} F-S c\left(\frac{2 n}{m+1}\right) H F
\end{aligned}
$$

At each iteration step, the system of linear partial differential equations (21) - (23) were expressed in finite difference form using central difference scheme in the $\eta$ - direction and backward difference scheme in $\xi$ direction. The resulting equations were then reduced to a system of linear algebraic equations with a block tri-diagonal matrix which is solved by using Varga's algorithm (Varga, 2000).

To ensure the convergence of the numerical solution to the exact solution, the step sizes $\Delta \eta$ and $\Delta \xi$ are optimized and taken as 0.01 and 0.005 respectively. The results presented here are independent of the step sizes at least up to the fifth decimal place. Convergence criteria based on the relative difference between the current and previous iteration values of the velocity, temperature and concentration gradients at the wall are employed. When the difference reaches less than $10^{-5}$, the solution is assumed to have converged and the iterative process is terminated.

\section{RESULTS AND DISCUSSION}

The effects of various physical parameters on the flow are examined and discussed in this section. The computations have been carried out for different values of parameters $A, m, n, \operatorname{Pr}, \lambda, S, M, K, S c$ and $\Delta$. In all the numerical computations, the edge of the boundary layer $\eta_{\infty}$ is taken as 6 . In order to verify the accuracy of our numerical method, the present results are compared with the existing theoretical results in literature. The results are found in very good agreement and the comparison is shown in Tables 1-3.

Table 1. Comparison of rate of heat transfer $\left(-\left(G_{\eta}\right)_{w}\right)$ results with Watanabe (1990), when $A=0, m=0.0909, n=0, \lambda=0, M=0$, $K=0, S c=0, \Delta=0$ and $\xi=0$.

\begin{tabular}{lcc}
\hline $\operatorname{Pr}$ & \multicolumn{1}{c}{$-\left(G_{\eta}\right)_{w}$} & \\
\cline { 2 - 3 } & Present results & Watanabe (1990) \\
\hline 0.3 & 0.32088 & 0.31967 \\
0.5 & 0.38849 & 0.38841 \\
0.73 & 0.44740 & 0.44730 \\
1 & 0.50200 & 0.50198 \\
2 & 0.64372 & 0.64372 \\
3 & 0.74251 & 0.74253 \\
5 & 0.88696 & 0.88706 \\
7 & 0.99626 & 0.99634 \\
10 & 1.12619 & 1.12618 \\
15 & 1.29357 & 1.29360 \\
\hline
\end{tabular}

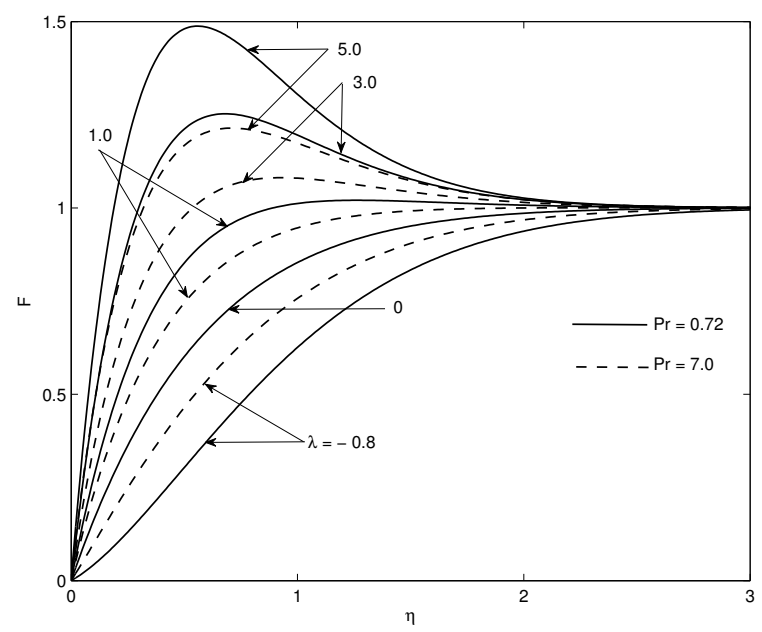

Fig. 2 Effects of $\operatorname{Pr}$ and $\lambda$ on velocity profiles when $A=0.5, m=$ $0.0909, n=1.0, S=1.0, M=0.1, K=0.1, S c=0.62, \Delta=$ $0.5, \xi=1.0$ and $\omega^{*}=\pi$. Slot position $\left[\xi_{0}=0.5, \xi_{0}^{*}=1.0\right]$.

Figures 2 and 3 display the effects of buoyancy force parameter $\lambda$ and the Prandtl number $\operatorname{Pr}$ on velocity and temperature profiles $(F, G)$. Both the buoyancy assisting $(\lambda>0)$ and opposing $(\lambda<0)$ flow cases are considered here. The buoyancy assisting flow shows overshoot in the velocity profiles near the wall for lower Prandtl number fluid $(\operatorname{Pr}=0.72$, air). The physical reason is that the buoyancy force $\lambda$ effect is larger in lower Prandtl number fluid due to low viscosity of the fluid, which enhances the velocity within the boundary layer as the assisting buoyancy force acts like a favorable pressure gradient and hence, the velocity overshoot occurs. The velocity overshoot is not observed for higher Prandtl number fluid ( $\mathrm{Pr}=7.0$, water) because water has more viscosity than air and more viscous fluid has less impact on the buoyancy force parameter. It is also observed that the velocity overshoot increases with the increase of buoyancy assisting force $(\lambda>0)$. There is no velocity overshoot for the buoyancy opposing flow $(\lambda<0)$. The reason is that the buoyancy opposed flow gives rise to an adverse pressure gradient which reduces the forced convection velocity and hence the velocity overshoot is not observed for opposing flow. It is noticed in Fig. 3 that the thermal boundary layer thickness is reduced with the increase of buoyancy 
Table 2. Comparison of skin friction parameter $\left(\left(F_{\eta}\right)_{w}\right)$ with those of Watanabe (1990), Watanabe et al. (1994), Yih (1998a), Ishak et al. (2007) and Kumari et al. (2001) when $\operatorname{Pr}=0.73, A=0, n=0, \lambda=0, M=0, K=0, S c=0, \Delta=0$ and $\xi=0$.

\begin{tabular}{ccccccc}
\hline \multirow{2}{*}{$m$} & \multicolumn{5}{c}{$\left(F_{\eta}\right)_{w}$} \\
\cline { 2 - 7 } & Present results & Watanabe (1990) & Watanabe et al. (1994) & Yih (1998a) & Ishak et al. (2007) & Kumari et al. (2001) \\
\hline 0 & 0.46969 & 0.46960 & 0.46960 & 0.469600 & 0.4696 & 0.46975 \\
0.0141 & 0.50480 & 0.50461 & - & 0.504614 & 0.5046 & 0.50472 \\
0.0435 & 0.56889 & 0.56898 & 0.56898 & 0.568978 & 0.5690 & 0.56904 \\
0.0909 & 0.65489 & 0.65498 & 0.65498 & 0.654979 & 0.6550 & 0.65501 \\
0.1429 & 0.73193 & 0.73200 & 0.73200 & 0.731998 & 0.7320 & 0.73202 \\
0.2000 & 0.80210 & 0.80215 & 0.80213 & 0.802125 & 0.8021 & 0.80214 \\
0.3333 & 0.92772 & 0.92765 & 0.92765 & 0.927653 & 0.9277 & 0.92766 \\
0.5000 & 1.03889 & - & 1.03890 & - & - & - \\
\hline
\end{tabular}

Table 3. Comparison of heat transfer parameter $\left(-\left(G_{\eta}\right)_{w}\right)$ with those of Watanabe (1990), Watanabe et al. (1994), Kumari et al. (1995) and Kumari et al. (2001) when $\operatorname{Pr}=0.73, A=0, n=0, \lambda=0, M=0, K=0, S c=0, \Delta=0$ and $\xi=0$.

\begin{tabular}{cccccc}
\hline \multirow{2}{*}{$m$} & \multicolumn{5}{c}{$-\left(G_{\eta}\right)_{w}$} \\
\cline { 2 - 6 } & Present results & Watanabe (1990) & Watanabe et al. (1994) & Kumari et al. (1995) & Kumari et al. $(2001)$ \\
\hline 0 & 0.42016 & 0.42015 & 0.42015 & 0.42014 & 0.42079 \\
0.0141 & 0.42585 & 0.42578 & - & 0.42579 & 0.42635 \\
0.0435 & 0.43556 & 0.43548 & 0.43548 & 0.43546 & 0.43597 \\
0.0909 & 0.44742 & 0.44730 & 0.44730 & 0.44732 & 0.44770 \\
0.1429 & 0.45707 & 0.45693 & 0.45693 & 0.45696 & 0.45728 \\
0.2000 & 0.46518 & 0.46503 & 0.46503 & 0.46505 & 0.46534 \\
0.3333 & 0.47820 & 0.47814 & 0.47814 & 0.47817 & 0.47840 \\
0.5000 & 0.48848 & - & 0.48848 & - & - \\
\hline
\end{tabular}

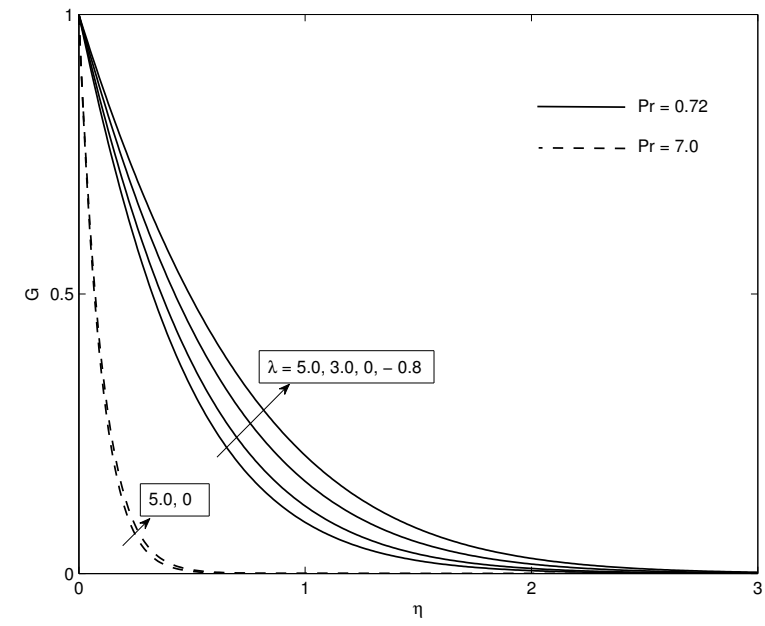

Fig. 3 Effects of $\operatorname{Pr}$ and $\lambda$ on temperature profiles when $A=0.5, m=$ $0.0909, n=1.0, S=1.0, M=0.1, K=0.1, S c=0.62, \Delta=$ $0.5, \xi=1.0$ and $\omega^{*}=\pi$. Slot position $\left[\xi_{0}=0.5, \xi_{0}^{*}=1.0\right]$.

parameter $\lambda$. Moreover, the thermal boundary layer thickness decreases as the Prandtl number increases. The physical reason is that the high Prandtl number fluid ( $\mathrm{Pr}=7.0$, water) means the momentum diffuses very quickly compared to the heat. This means that the thermal boundary layer is very thin relative to the velocity boundary layer. Therefore, high Prandtl number fluid result in thinner thermal boundary layer.

Figure 4 depicts the dimensionless velocity and temperature profiles $(F, G)$ for different values of suction parameter $(A>0)$ and injection parameter $(A<0)$, respectively. It is observed that the velocity component of the fluid along the wall of the wedge increases with increase of suction

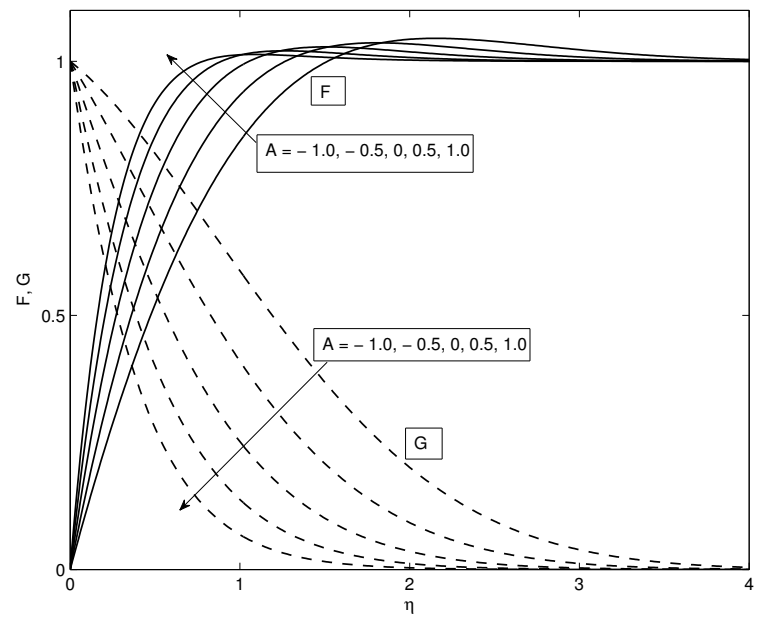

Fig. 4 Effects of suction $(A>0)$ and injection $(A<0)$ on velocity and temperature profiles when $m=0.0909, n=1.0, \operatorname{Pr}=0.72$, $\lambda=1.0, S=1.0, M=0.1, K=0.1, S c=0.62, \Delta=0.5$, $\xi=1.0$ and $\omega^{*}=\pi$. Slot position $\left[\xi_{0}=0.5, \xi_{0}^{*}=1.0\right]$.

and decreases with increase of injection at the wall of the wedge. On the contrary, the dimensionless temperature of the fluid reduces with increase of suction and increases with increase of injection. Therefore, the increase of suction accelerates the fluid motion and decreases the temperature distribution of the fluid along the wall of the wedge. On the other hand, the increase of injection decelerates the fluid motion and increases the temperature distribution of the fluid along the wall of the wedge.

The effect of the Schmidt number $S c$ on concentration profiles is 


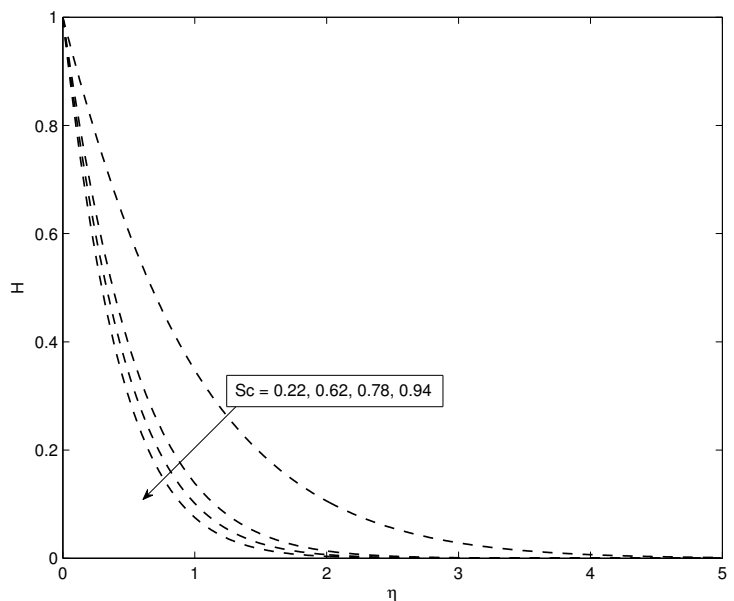

Fig. 5 Effect of $S c$ on concentration profiles when $A=0.5, m=0.0909$, $n=1.0, P r=0.72, \lambda=1.0, S=1.0, M=1.0, K=0.5$, $\Delta=0.0, \xi=1.0$ and $\omega^{*}=\pi$. Slot position $\left[\xi_{0}=0.5, \xi_{0}^{*}=\right.$ $1.0]$.

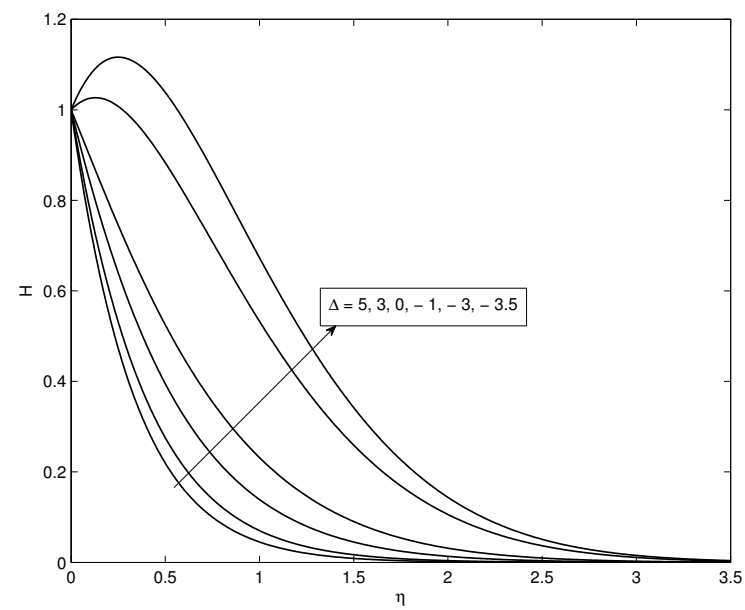

Fig. 6 Effect of $\Delta$ on concentration profiles when $A=0.5, m=0.0909$, $n=1.0, P r=0.72, \lambda=1.0, S=1.0, M=1.0, K=0.5$, $S c=0.62, \xi=1.0$ and $\omega^{*}=\pi$. Slot position $\left[\xi_{0}=0.5, \xi_{0}^{*}=\right.$ 1.01 .

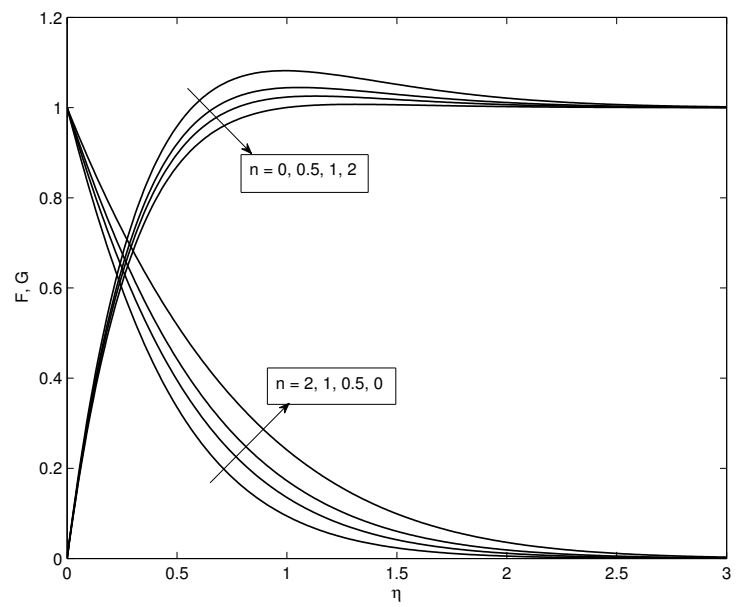

Fig. 7 Effect of $n$ on velocity and temperature profiles when $A=0.5$, $m=0.0909, P r=0.72, \lambda=1.0, S=1.0, M=1.0, K=$ $0.5, S c=0.62, \Delta=0.5, \xi=1.0$ and $\omega^{*}=\pi$. Slot position $\left[\xi_{0}=0.5, \xi_{0}^{*}=1.0\right]$.

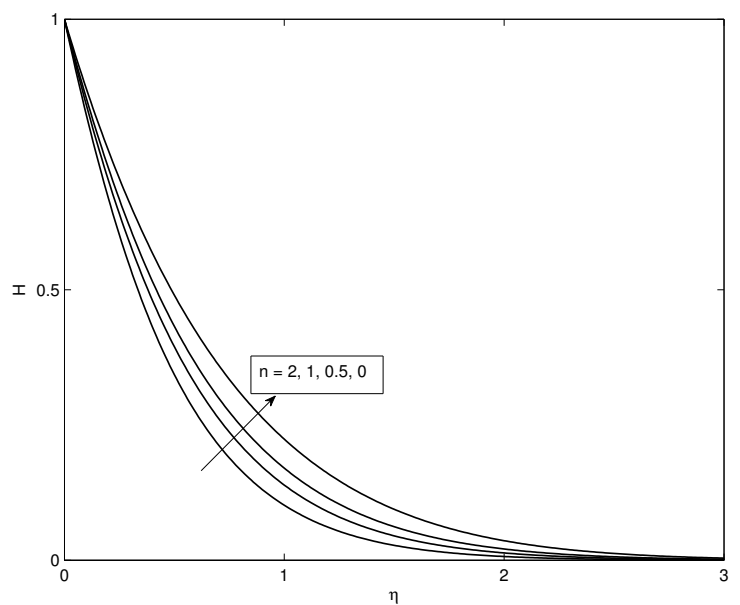

Fig. 8 Effect of $n$ on concentration profiles when $A=0.5, m=0.0909$, $P r=0.72, \lambda=1.0, S=1.0, M=1.0, K=0.5, S c=0.62$, $\Delta=0.5, \xi=1.0$ and $\omega^{*}=\pi$. Slot position $\left[\xi_{0}=0.5, \xi_{0}^{*}=\right.$ 1.01 .

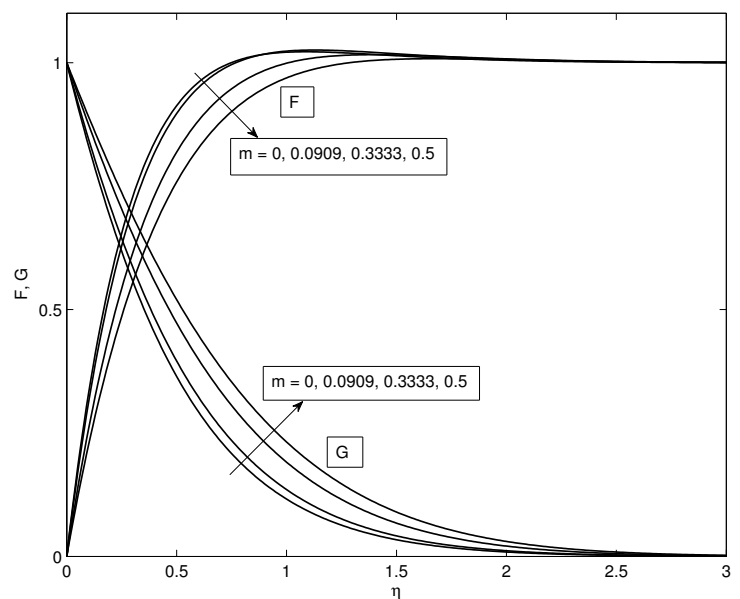

Fig. 9 Effect of $m$ on velocity and temperature profiles when $A=0.5$, $n=1.0, \operatorname{Pr}=0.72, \lambda=1.0, S=1.0, M=1.0, K=0.5$, $S c=0.62, \Delta=0.5, \xi=1.0$ and $\omega^{*}=\pi$. Slot position $\left\lceil\xi_{0}=0.5, \xi_{0}^{*}=1.0\right\rceil$.

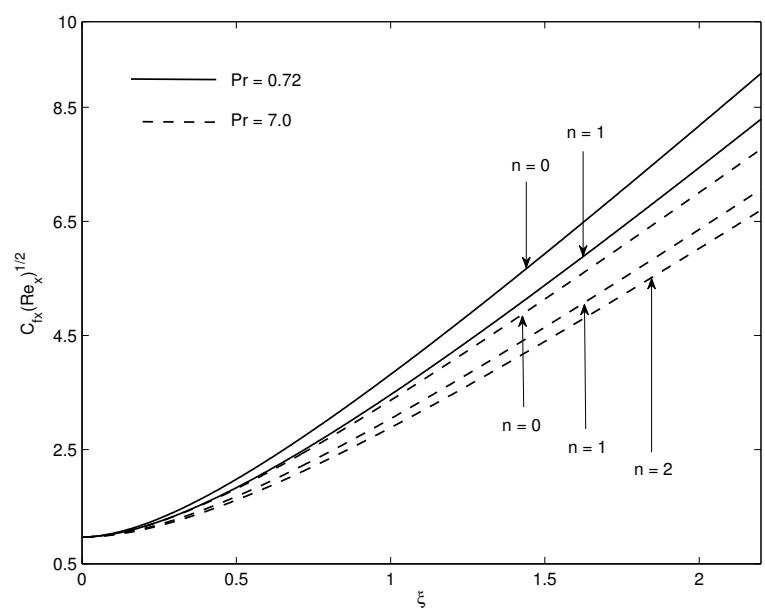

Fig. 10 Effects of $P r$ and $n$ on $C_{f x}\left(R e_{x}\right)^{1 / 2}$ when $A=0.0, m=$ $0.0909, \lambda=1.0, S=1.0, M=0.1, K=0.1, S c=0.62$ and $\Delta=0.5$ 
shown in Fig. 5. The values of Schmidt number are chosen to be realistic, hydrogen $(S c=0.22)$, water vapor $(S c=0.62)$, ammonia $(S c=0.78)$ and hydrogen sulphide $(S c=0.94)$ at $25^{\circ} \mathrm{C}$ and at one atmospheric pressure. It is seen from figure that an increase in $S c$ causes a reduction in the concentration boundary layer thickness. The physical reason is that the high value of $S c$ has a low mass diffusivity which leads to a thinning of the concentration boundary layer.

Effects of chemical reaction in the presence of uniform magnetic field play an important role in the concentration field. Figure 6 depicts the variations of $\Delta$ on concentration $H$. It is evident that concentration $H$ enhances for larger species consumption parameter $(\Delta<0)$. However, concentration $H$ has opposite effects for species generation parameter $(\Delta>0)$. Physically larger values of species generation parameter correspond to higher rate of generative chemical reaction which generates the fluid specie more efficiently and therefore, concentration distribution increases. However, reverse situation is observed for species consumption parameter $(\Delta<0)$. Moreover, the concentration boundary layer thickness is reduced by species generation and it is opposite for species consumption. The physical reason is that the presence of species generation effect has the tendency to increase the concentration state of the fluid causing its concentration and concentration boundary layer to decrease. Figures 7 and 8 show the representative velocity, temperature and concentration profiles for different values of the wall temperature or concentration index $n$ when $A=0.5, m=0.0909, \operatorname{Pr}=0.72, \lambda=1.0$, $S=1.0, M=1.0, K=0.5, S c=0.62, \Delta=0.5, \xi=1.0$ and $\omega^{*}=\pi$. It is clearly observed that both the fluid temperature and solute concentration decrease as $n$ increases. This yields enhancements in both heat and mass transfer effects. The power index $m$ in the free stream velocity has different effects on velocity and temperature inside the boundary layer. Figure 9 illustrates the effects of the variable power law of the free stream velocity on velocity and temperature profiles. It is seen that an increase in $m$ decreases the fluid velocity and temperature inside the boundary layer.

The effect of Prandtl number $\operatorname{Pr}$ on the skin friction coefficient $\left(C_{f x}\left(R e_{x}\right)^{1 / 2}\right)$ and Nusselt number $\left(N u_{x}\left(R e_{x}\right)^{-1 / 2}\right)$ is shown in Figs. 10 and 11. It is evident from figure 11 that the heat transfer rate or Nusselt number enhances with $\mathrm{Pr}$. The reason is that the high Prandtl number fluid result in thinner thermal boundary layer and hence, a higher heat transfer rate at the wall. Also, it is noted that the skin friction coefficient is decreased by increasing $P r$. The physical reason is that water $(\operatorname{Pr}=7.0)$ has more viscosity than air $(\operatorname{Pr}=0.72)$, and more viscous fluid which increases the boundary layer thickness and consequently, reduces the wall shear stress and the skin friction coefficient. The Sherwood number $\left(S h_{x}\left(R e_{x}\right)^{-1 / 2}\right)$ enhances with $S c$ which can be seen in Fig. 12. The effect of variable wall temperature (or concentration) index $n$ on the skin friction coefficient, Nusselt and Sherwood numbers is determined in Figs. 10-12 respectively. It is found that Nusselt and Sherwood numbers enhance with $n$, while the skin friction coefficient is reduced by increasing $n$.

Figures 13-15 illustrate the effects of suction $(A>0)$ and injection $(A<0)$ on skin friction coefficient $\left(C_{f x}\left(R e_{x}\right)^{1 / 2}\right)$, Nusselt number $\left(N u_{x}\left(R e_{x}\right)^{-1 / 2}\right)$ and Sherwood number $\left(S h_{x}\left(R e_{x}\right)^{-1 / 2}\right)$. It is observed that the skin friction, heat and mass transfer coefficients are decreased by an increasing of injection parameter. The reason is that the thickness of the velocity, thermal and concentration boundary layers grow with the increase of injection parameter. Consequently, the wall shear stress and, the rate of heat and mass transfer at the wall reduce considerably. Thus, the heat and mass transfer rates can be reduced by increasing of injection.

The effect of chemical reaction parameter $\Delta(\Delta>0$ for species generation, $\Delta<0$ for species consumption and $\Delta=0$ for no chemical reaction) on the Sherwood number is shown in Fig. 16. It is found that the Sherwood number increases with species generation, while decreases with species consumption. The physical reason is that the presence of

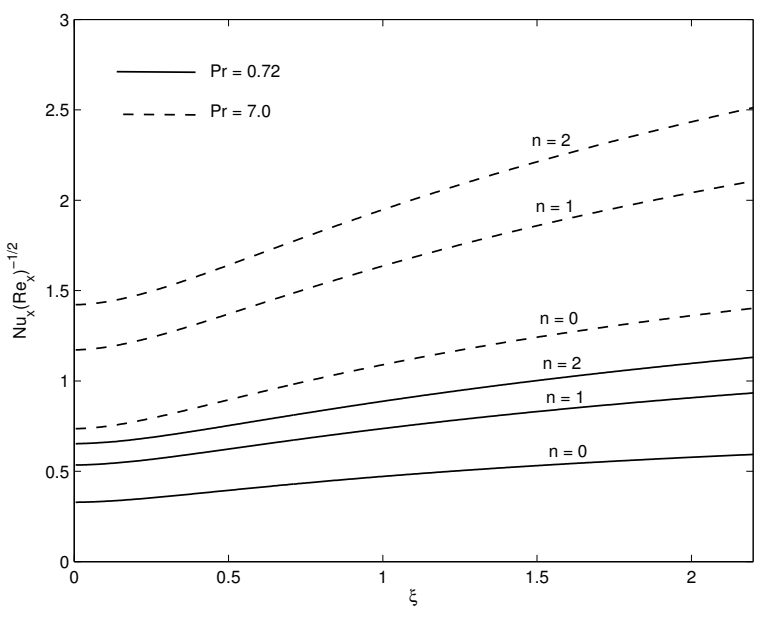

Fig. 11 Effects of $\operatorname{Pr}$ and $n$ on $N u_{x}\left(R e_{x}\right)^{-1 / 2}$ when $A=0.0, m=$ $0.0909, \lambda=1.0, S=1.0, M=0.1, K=0.1, S c=0.62$ and $\Delta=0.5$.

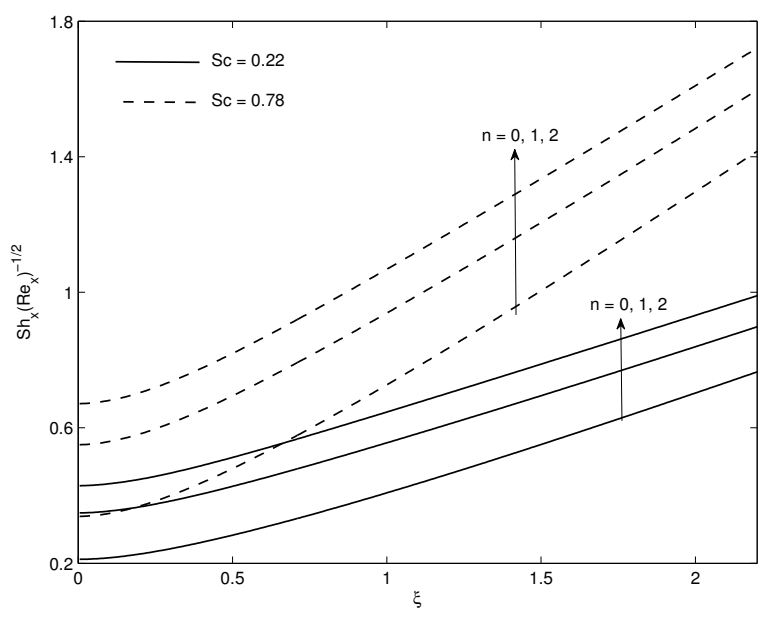

Fig. 12 Effects of $S c$ and $n$ on $S h_{x}\left(R e_{x}\right)^{-1 / 2}$ when $A=0.0, m=$ $0.0909, \operatorname{Pr}=0.72, \lambda=1.0, S=1.0, M=0.1, K=0.1$ and $\Delta=0.5$.

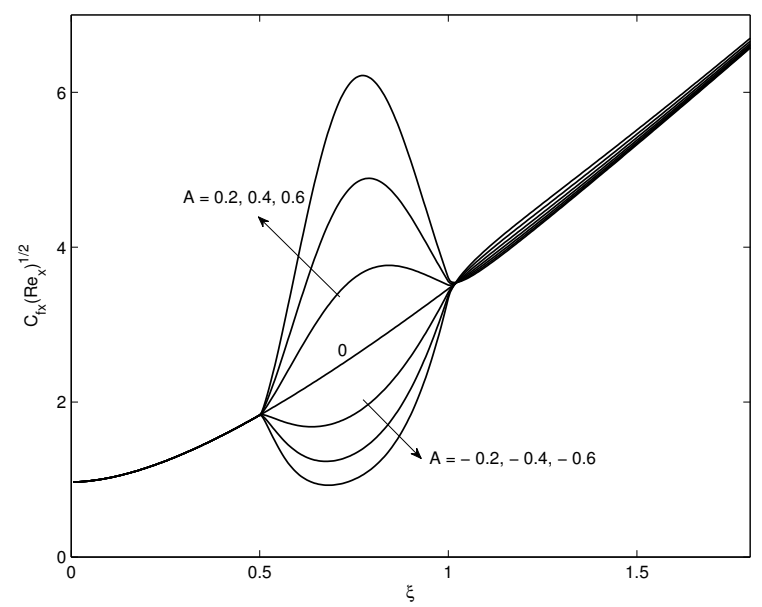

Fig. 13 Effects of suction $(A>0)$ and injection $(A<0)$ on $C_{f x}\left(R e_{x}\right)^{1 / 2}$ when $m=0.0909, n=1.0, \operatorname{Pr}=0.72, \lambda=1.0$, $S=1.0, M=0.1, K=0.1, S c=0.62, \Delta=0.5$ and $\omega^{*}=2 \pi$. Slot position $\left[\xi_{0}=0.5, \xi_{0}^{*}=1.0\right]$. 


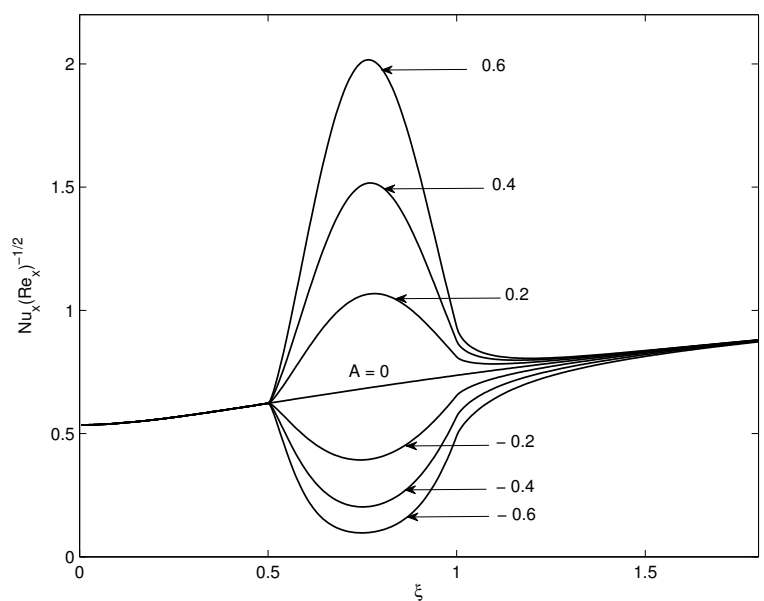

Fig. 14 Effects of suction $(A>0)$ and injection $(A<0)$ on $N u_{x}\left(R e_{x}\right)^{-1 / 2}$ when $m=0.0909, n=1.0, P r=0.72$, $\lambda=1.0, S=1.0, M=0.1, K=0.1, S c=0.62, \Delta=0.5$ and $\omega^{*}=2 \pi$. Slot position $\left\lceil\xi_{0}=0.5, \xi_{n}^{*}=1.0\right\rceil$.

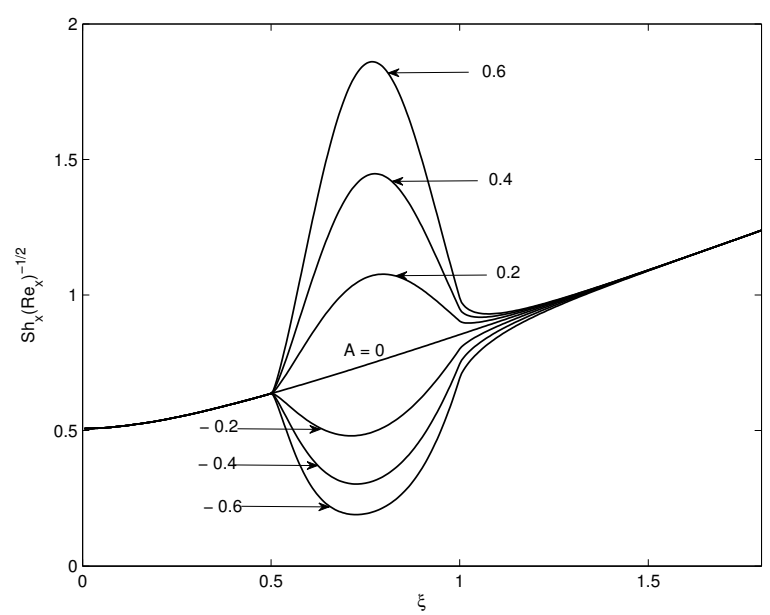

Fig. 15 Effects of suction $(A>0)$ and injection $(A<0)$ on $S h_{x}\left(R e_{x}\right)^{-1 / 2}$ when $m=0.0909, n=1.0, \operatorname{Pr}=0.72$, $\lambda=1.0, S=1.0, M=0.1, K=0.1, S c=0.62, \Delta=0.5$ and $\omega^{*}=2 \pi$. Slot position $\left\lceil\xi_{0}=0.5, \xi_{n}^{*}=1.0\right\rceil$.

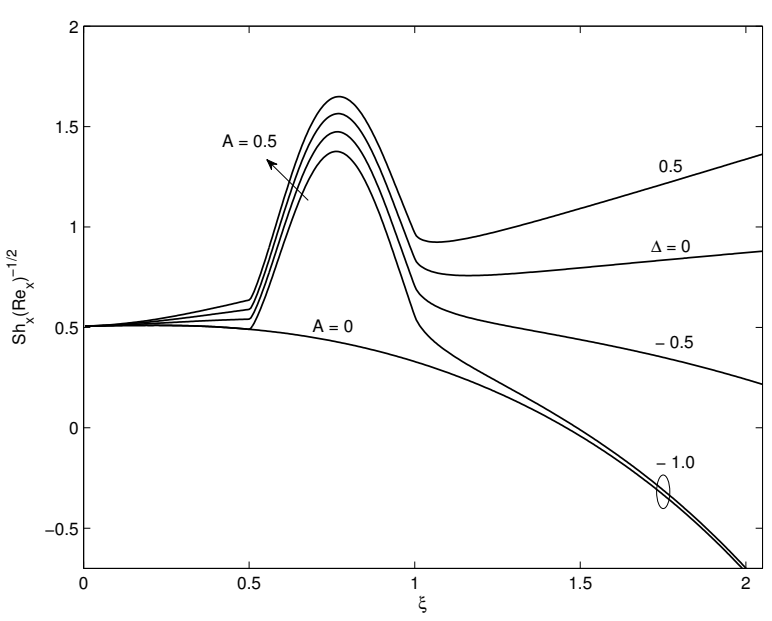

Fig. 16 Effect of $\Delta$ on $S h_{x}\left(R e_{x}\right)^{-1 / 2}$ when $m=0.0909, n=1.0$, $\operatorname{Pr}=0.72, \lambda=1.0, S=1.0, M=0.1, K=0.1, S c=0.62$ and $\omega^{*}=2 \pi$. Slot position $\left[\xi_{0}=0.5, \xi_{0}^{*}=1.0\right]$.

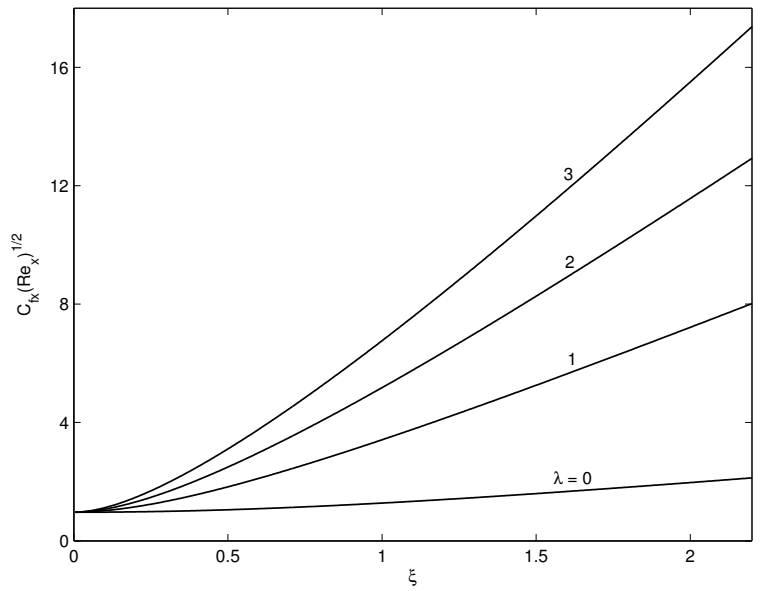

Fig. 17 Effect of $\lambda$ on $C_{f x}\left(R e_{x}\right)^{1 / 2}$ when $A=0.0, m=0.0909$, $n=1.0, P r=0.72, S=1.0, M=0.1, K=0.1, S c=0.62$ and $\Delta=1.0$.

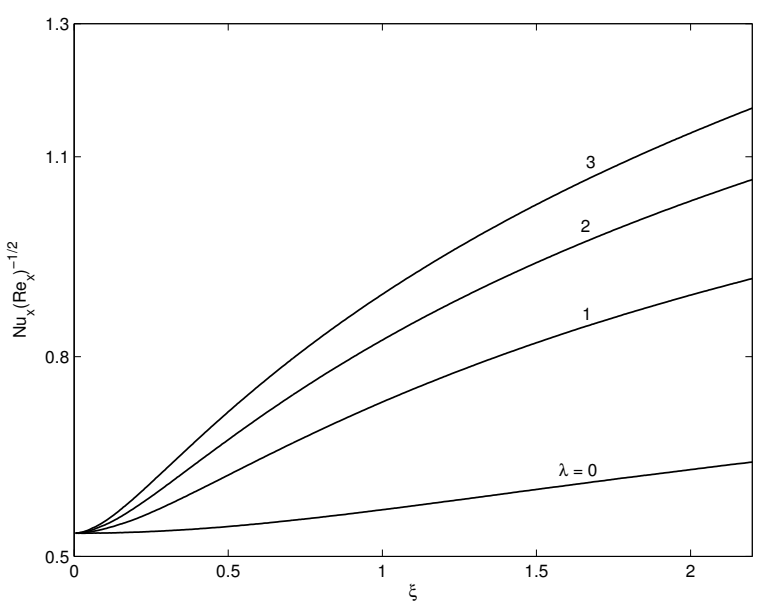

Fig. 18 Effect of $\lambda$ on $N u_{x}\left(R e_{x}\right)^{-1 / 2}$ when $A=0.0, m=0.0909$, $n=1.0, P r=0.72, S=1.0, M=0.1, K=0.1, S c=0.62$ and $\Delta=1.0$.

species generation effect has the tendency to decrease the concentration state of the fluid causing its concentration and concentration boundary layer to decrease, and consequently, the negative concentration gradient and hence, the Sherwood number increases with species generation. On the other hand, when the species consumption effects are present, the reverse trends where both the fluid concentration and its concentration boundary layer to increase, and consequently, the positive concentration gradient and hence, the mass transfer rate or Sherwood number decreases with species consumption. The negative values in the mass transfer rate are due to large species consumption effects.

The effect of buoyancy force parameter $\lambda$ on skin friction coefficient and Nusselt number is shown in Figs. 17 and 18. It is observed from figures both the skin friction coefficient and Nusselt number are enhanced with $\lambda$. The reason for such behaviour is that the positive buoyancy parameter $(\lambda>0)$ acts like a favorable pressure gradient and the fluid gets accelerated, which results in thinner momentum and thermal boundary layers, and consequently, the skin friction coefficient and Nusselt number are increased. The skin friction coefficient is more pronounced than the Nusselt number due to buoyancy.

Figures 19 and 20 display the effect of magnetic parameter $M$ on the skin friction coefficient and Nusselt number. The skin friction coef- 


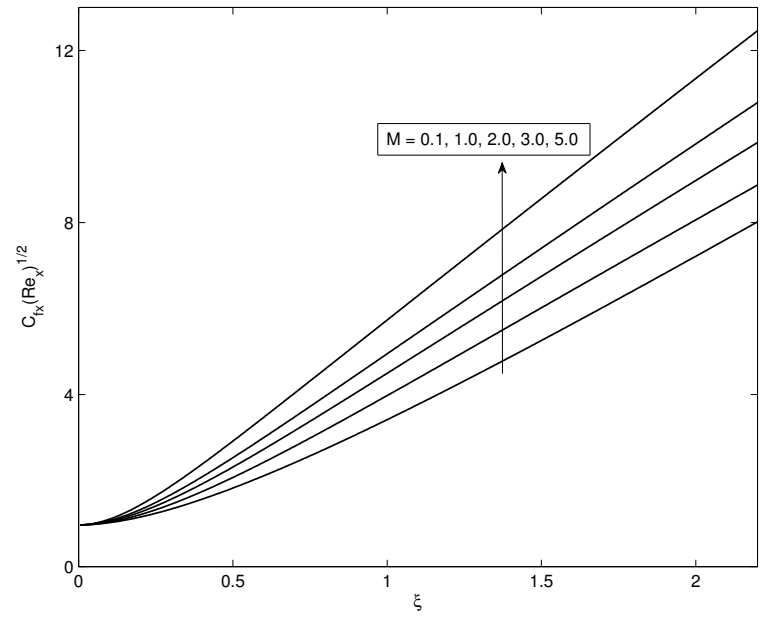

Fig. 19 Effect of $M$ on $C_{f x}\left(R e_{x}\right)^{1 / 2}$ when $A=0.0, m=0.0909$, $n=1.0, \operatorname{Pr}=0.72, \lambda=1.0, S=1.0, K=0.1, S c=0.62$ and $\Delta=1.0$.

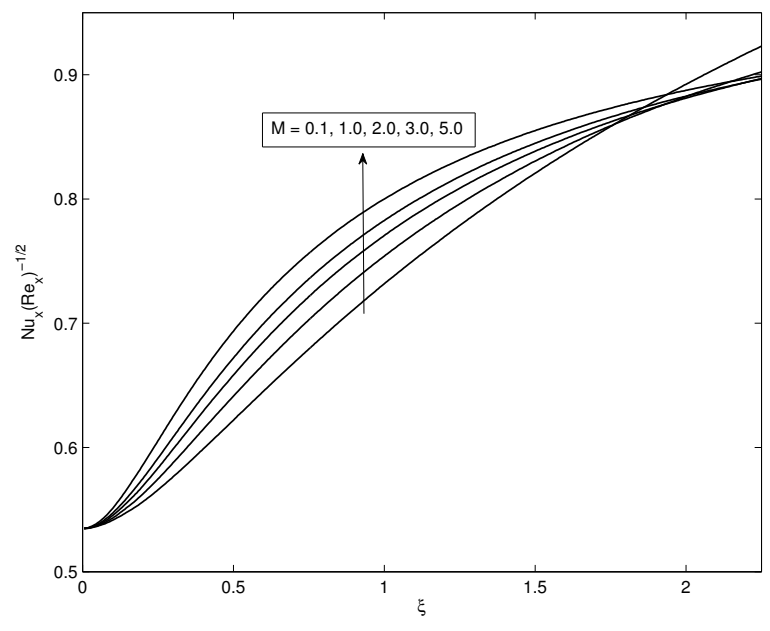

Fig. 20 Effect of $M$ on $N u_{x}\left(R e_{x}\right)^{-1 / 2}$ when $A=0.0, m=0.0909$, $n=1.0, \operatorname{Pr}=0.72, \lambda=1.0, S=1.0, K=0.1, S c=0.62$ and $\Delta=1.0$.

ficient increases with $M$ due to enhanced Lorentz force which imports an additional momentum in the boundary layer. It is also observed that the effect of magnetic parameter $M$ on the skin friction coefficient and Nusselt number becomes more pronounced as the distance $\xi$ increases. The Nusselt number increases as $M$ increases until certain $\xi$, say $\xi^{*}$. For $\xi>\xi^{*}$, Nusselt number is decreased by increasing $M$. The reason is that after certain $\xi$, a greater retarding effect is generated in the flow with greater $M$ values (i.e. stronger magnetic field strengths) which causes the prominent depression in Nusselt number.

\section{CONCLUSIONS}

The effects of chemical reaction and non-uniform slot suction or injection into MHD mixed convective heat and mass transfer flow over a vertical wedge embedded in a porous medium have been analyzed by non-similar solutions for the case of variable wall temperature and concentration. The main conclusions of the present study are as follows:

- The heat transfer and temperature field are strongly influenced by the Prandtl number.
- The concentration boundary layer thickness is decreased by the increase of species generation and Schmidt number.

- The assisting buoyancy force is found to cause overshoot in the velocity profiles for lower Prandtl number fluids.

- The skin friction and heat transfer coefficients are increased by the increase of buoyancy parameter.

- The local skin friction coefficient, the local Nusselt number, and the local Sherwood number will increase when suction is present at the permeable wall, whereas the opposite trend is true for the case when the wall is subjected to injection of fluid.

- The local Nusselt number can be increased by increasing the values of the Prandtl number and the wall temperature/concentration exponent, whereas the local skin friction coefficient is decreased by increasing the values of the Prandtl number and the wall temperature/concentration exponent. In addition, increasing both the Schmidt number and the power-law exponent will produce an increase in the local Sherwood number.

- The skin friction coefficient is strongly affected by the magnetic parameter, whereas the heat transfer coefficient weakly dependent on the magnetic parameter.

Viscous dissipation and Joule heating effects, unsteady mixed convective flow and different fluids can be considered which may have significant impacts on the fluid flow and, heat and mass transfer characteristics for the flow along a wedge in porous medium.

\section{ACKNOWLEDGEMENTS}

Authors are thankful to the reviewers for their valuable comments and constructive suggestions to improve the quality of the manuscript. One of the authors Ganapathirao wish to thank to Dr. Vaishali Shinde for her help in English language check in the manuscript.

\section{NOMENCLATURE}

$A \quad$ surface mass transfer parameter

$b_{1}, b_{2} \quad$ constants

$B \quad$ magnetic field induction

$c_{p} \quad$ specific heat at a constant pressure $\left(\mathrm{kJ} \cdot \mathrm{kg}^{-1} \cdot \mathrm{K}^{-1}\right)$

C species concentration

$C_{f x} \quad$ local skin friction coefficient

$D \quad$ mass diffusivity $\left(\mathrm{m}^{2} \cdot \mathrm{s}^{-1}\right)$

$f \quad$ dimensionless stream function

$F \quad$ dimensionless velocity

$g \quad$ acceleration due to gravity $\left(\mathrm{m} \cdot \mathrm{s}^{-2}\right)$

$G \quad$ dimensionless temperature

$G r_{L}, G r_{L}^{*}$ Grashof numbers due to temperature and concentration, respectively

$H \quad$ dimensionless concentration

$\mathrm{Ha}$ Hartmann number

$k_{c} \quad$ chemical reaction rate $\left(\mathrm{s}^{-1}\right)$

$K \quad$ dimensionless permeability parameter

$K_{1}, K_{2} \quad$ dimensional and dimensionless permeability parameters, respectively

$L \quad$ characteristic length (m)

$m \quad$ index in the power-law variation of the velocity at the edge of the boundary layer

$n \quad$ index in the power-law variation of the temperature and concentration at the edge of the boundary layer

$M \quad$ magnetic parameter

$N u_{x} \quad$ local Nusselt number

$\mathrm{Pr} \quad$ Prandtl number 


$\begin{array}{ll}R e_{L}, R e_{x} & \begin{array}{l}\text { Reynolds numbers defined with respect to } L \text { and } x, \\ \text { respectively }\end{array} \\ S & \text { ratio of the buoyancy forces or ratio of Grashof numbers } \\ S c & \begin{array}{l}\text { Schmidt number } \\ \text { dimensional temperature }(\mathrm{K})\end{array} \\ T & \text { velocity components along } x \text { and } y \text { - directions, } \\ u, v & \text { respectively }\left(\mathrm{m} \cdot \mathrm{s}^{-1}\right)\end{array}$

\section{REFERENCES}

Bellman, R. E. and Kalaba. R. E., 1965, Quasilinearization and Nonlinear Boundary-Value Problems, USA, Elsevier.

Chamkha, A. J., 1996, "Non-Darcy Hydromagnetic Free Convection from a Cone and a Wedge in Porous Media," International Communications in Heat and Mass Transfer, 23, 875-887. http://dx.doi.org/10.1016/0735-1933(96)00070-X

Chamkha, A. J., Khaled, A. -R. A., and Al-Hawaj, O., 2000, "Simultaneous Heat and Mass Transfer by Natural Convection from a Cone and a Wedge in Porous Media," Journal of Porous Media, 3, 155-164. http://dx.doi.org/10.1615/JPorMedia.v3.i2.50

Chamkha, A. J., Takhar, H. S., and Nath, G., 2004, "Mixed Convection Flow over a Vertical Plate with Localized Heating (Cooling), Magnetic Field and Suction (Injection)," Heat and Mass Transfer, 40, 835-841. http://dx.doi.org/10.1007/s00231-003-0465-5

Datta, P., Anilkumar, D., Roy, S., and Mahanti, N. C., 2006, "Effect of Non-uniform Slot Injection (Suction) on a Forced Flow over a Slender Cylinder," International Journal of Heat and Mass Transfer, 49, 23662371. http://dx.doi.org/10.1016/j.ijheatmasstransfer.2005.10.044

Devi, S. P. A. and Kandasamy, R., 2002, "Effects of Chemical Reaction, Heat and Mass Transfer on Non-linear MHD Laminar Boundary-layer Flow over a Wedge with Suction or Injection," International Communications in Heat and Mass Transfer, 29, 707-716. http://dx.doi.org/10.1016/S0735-1933(02)00389-5

Ganapathirao, M., Revathi, G., and Ravindran, R., 2014, "Unsteady Mixed Convection Boundary Layer Flow over a Vertical Cone with Non-uniform Slot Suction (Injection)," Meccanica, 49, 673-686. http://dx.doi.org/10.1007/s11012-013-9819-3
Gorla, R. S. R, Chamkha, A. J., Khan, W. A., and Murthy, P. V. S. N., 2012, "Second Law Analysis for Combined Convection in Non-Newtonian Fluids over a Vertical Wedge Embedded in a Porous Medium," Journal of Porous Media, 15, 187-196. http://dx.doi.org/10.1615/JPorMedia.v15.i2.70

Inouye, K. and Tate, A., 1974, "Finite-difference Version of Quasilinearization Applied to Boundary-layer Equations," AIAA Journal, 12, $558-560$.

http://dx.doi.org/10.2514/3.49286

Ishak, A., Nazar, R., and Pop, I., 2007, "Falkner-Skan Equation for Flow past a Moving Wedge with Suction or Injection," Journal of Applied Mathematics and Computing, 25, 67-83. http://dx.doi.org/10.1007/BF02832339

Kafoussias, N. G. and Nanousis, N. D., 1997, "Magnetohydrodynamic Laminar Boundary-layer Flow over a Wedge with Suction or Injection," Canadian Journal of Physics, 75, 733-754. http://dx.doi.org/10.1139/p97-024

Kandasamy, R., Hashim, I., and Muhaimin, 2008, "Chemical Reaction and Variable Viscosity Effects on MHD Mixed Convection Heat and Mass Transfer for a Hiemenz Flow over a Porous Wedge in the Presence of Suction or Injection," International Journal of Fluid Mechanics Research, 35, 1-18. http://dx.doi.org/10.1615/InterJFluidMechRes.v35.i1.10

Kandasamy, R., Nordin, M., and Khamis, A. B., 2010, "Variable Viscosity and Chemical Reaction Effects on Non-Darcy Magnetohydrodynamic Mixed Convective Heat and Mass Transfer past a Porous Wedge in the Presence of Suction or Injection,' Journal of Porous Media, 13, 579-590. http://dx.doi.org/10.1615/JPorMedia.v13.i6.80

Kandasamy, R., Periasamy, K., and Sivagnana Prabhu, K. K., 2005, "Effects of Chemical Reaction, Heat and Mass Transfer along a Wedge with Heat Source and Concentration in the Presence of Suction or Injection," International Journal of Heat and Mass Transfer, 48, 1388-1394. http://dx.doi.org/10.1016/j.ijheatmasstransfer.2004.10.008

Koh, J. C. Y. and Hartnett, J. P., 1961, "Skin Friction and Heat Transfer for Incomressible Laminar Flow over Porus Wedges with Suction and Variable Wall Temperature," International Journal of Heat and Mass Transfer, 2, 185-198.

http://dx.doi.org/10.1016/0017-9310(61)90088-6

Kumari, M. and Gorla, R. S. R., 1997, "Combined Convection along a Non-isothermal Wedge in a Porous Medium," Heat and Mass Transfer, 32, 393-398.

http://dx.doi.org/10.1007/s002310050136

Kumari, M., Takhar, H. S., and Nath, G., 1995, "Nonsimilar Mixed Convection Flow of a Non-Newtonian Fluid past a Vertical Wedge," Acta Mechanica, 113, 205-213.

http://dx.doi.org/10.1007/BF01212643

Kumari, M., Takhar, H. S., and Nath, G., 2001, "Mixed Convection Flow over a Vertical Wedge Embedded in a Highly Porous Medium," Heat and Mass Transfer, 37, 139-146.

http://dx.doi.org/10.1007/s002310000154

Minkowycz, W. J., Sparrow, E. M., Schneider, G. E. and Pletcher, R. H., 1988, Hand Book of Numerical Heat Transfer, New York, Wiley.

Muhaimin and Khamis, A. B., 2010, "Local Nonsimilarity Solution on MHD Convective Heat Transfer Flow past a Porous Wedge in the Presence of Suction/Injection," Journal of Porous Media, 13, 487-495. http://dx.doi.org/10.1615/JPorMedia.v13.i5.70 
Nanousis, N. D., 1999, "Theoretical Magnetohydrodynamic Analysis of Mixed Convection Boundary-layer Flow over a Wedge with Uniform Suction or Injection," Acta Mechanica, 138, 21-30. http://dx.doi.org/10.1007/BF01179539

Napolitano, M. and Messick, R. E., 1980, "On Strong Slot Injection into a Subsonic Laminar Boundary Layer," Computers \& Fluids, 8, 199-212. http://dx.doi.org/10.1016/0045-7930(80)90011-0

Ravindran, R. and Ganapathirao, M., 2013, "Non-uniform Slot Suction/Injection into Mixed Convection Boundary Layer Flow over Vertical Cone," Applied Mathematics and Mechanics, 34, 1327-1338. http://dx.doi.org/10.1007/s10483-013-1748-7

Riley, N., 1976, "Oblique Slot Blowing into a Supersonic Laminar Boundary Layer," Mathematical Proceedings of the Cambridge Philosophical Society, 80, 541-554.

http://dx.doi.org/10.1017/S0305004100053160

Riley, N., 1981, "Non-uniform Slot Injection into a Laminar Boundary Layer," Journal of Engineering Mathematics, 15, 299-314. http://dx.doi.org/10.1007/BF00042926

Roy, S. and Saikrishnan, P., 2003, "Non-uniform Slot Injection (Suction) into Steady Laminar Water Boundary Layer Flow over a Rotating Sphere," International Journal of Heat and Mass Transfer, 46, 33893396.

http://dx.doi.org/10.1016/S0017-9310(03)00137-6

Roy, S. and Saikrishnan, P., 2004, "Non-uniform Slot Injection (Suction) into Water Boundary Layer Flow past Yawed Cylinder," International Journal of Engineering Science, 42, 2147-2157. http://dx.doi.org/10.1016/j.ijengsci.2003.12.008

Saikrishnan, P. and Roy, S., 2003, "Non-uniform Slot Injection (Suction) into Water Boundary Layers over (i) a Cylinder and (ii) a Sphere," International Journal of Engineering Science, 41, 1351-1365. http://dx.doi.org/10.1016/S0020-7225(03)00043-0

Samyuktha, N., Ravindran, R., and Ganapathirao, M., 2016, "Unsteady Mixed Convection Flow over a Vertical Plate in a Porous Medium with Non-uniform Slot Suction/Injection," Journal of Porous Media, 19, 913930. http://dx.doi.org/10.1615/JPorMedia.v19.i10.60

Schlichting, H. and Gersten, K., 2000, Boundary-Layer Theory, New York, Springer.

Selimefendigil, F. and Öztop, H. F., 2018, "Magnetic Field Effects on the Forced Convection of CuO-Water Nanofluid Flow in a Channel with Circular Cylinders and Thermal Predictions using ANFIS," International Journal of Mechanical Sciences, 146-147, 9-24. http://dx.doi.org/10.1016/j.ijmecsci.2018.07.011

Selimefendigil, F. and Öztop, H. F., 2019a, "Fluid-Solid Interaction of Elastic-step type Corrugation Effects on the Mixed Convection of Nanofluid in a Vented Cavity with Magnetic Field," International Journal of Mechanical Sciences, 152, 185-197. http://dx.doi.org/10.1016/j.ijmecsci.2018.12.044

Selimefendigil, F. and Öztop, H. F., 2019b, "MHD Pulsating Forced Convection of Nanofluid over Parallel Plates with Blocks in a Channel," International Journal of Mechanical Sciences, 157-158, 726-740. http://dx.doi.org/10.1016/j.ijmecsci.2019.04.048

Smith, F. T. and Stewartson, K., 1973, "On Slot Injection into a Supersonic Laminar Boundary Layer," Proceedings of the Royal Society A, 332, 1-22. http://dx.doi.org/10.1098/rspa.1973.0010

Varga, R. S., 2000, Matrix Iterative Analysis, New York, Springer.
Watanabe, T., 1990, "Thermal Boundary Layers over a Wedge with Uniform Suction or Injection in Forced Flow," Acta Mechanica, 83, 119-126. http://dx.doi.org/10.1007/BF01172973

Watanabe, T., Funazaki, K., and Taniguchi, H., 1994, "Theoretical Analysis on Mixed Convection Boundary Layer Flow over a Wedge with Uniform Suction or Injection," Acta Mechanica, 105, 133-141. http://dx.doi.org/10.1007/BF01183947

Yih, K. A., 1998a, "Uniform Suction/Blowing Effect on Forced Convection about a Wedge: Uniform Heat Flux," Acta Mechanica, 128, 173-181. http://dx.doi.org/10.1007/BF01251888

Yih, K. A., 1998b, "Coupled Heat and Mass Transfer in Mixed Convection over a Wedge with Variable Wall Temperature and Concentration in Porous Media: The Entire Regime," International Communications in Heat and Mass Transfer, 25, 1145-1158. http://dx.doi.org/10.1016/S0735-1933(98)00105-5

Yih, K. A., 1999, "MHD Forced Convection Flow Adjacent to a Nonisothermal Wedge," International Communications in Heat and Mass Transfer, 26, 819-827. http://dx.doi.org/10.1016/S0735-1933(99)000706

\section{APPENDIX}

The steps involved in obtaining the non-dimensional equations (7)- (9) from the governing equations are as follows:

$$
\begin{aligned}
u_{e} & =u_{\infty}(\bar{x})^{m}, \quad \bar{x}=\frac{x}{L}, \quad m=\frac{\bar{x}}{u_{e}} \frac{d u_{e}}{d \bar{x}}=\frac{\gamma}{2-\gamma} \\
\eta_{y} & =\left(\frac{m+1}{2} \frac{u_{e}}{x \nu}\right)^{1 / 2}, \quad \eta_{x}=\left(\frac{m-1}{2 \bar{x}}\right) \eta \\
\frac{\partial \psi}{\partial y} & =\left(\frac{2}{m+1} x \nu u_{e}\right)^{1 / 2} f_{\eta} \eta_{y} \\
\frac{\partial \psi}{\partial x} & =\left(\frac{2 \nu u_{\infty}}{m+1}\right)^{1 / 2} \frac{\partial}{\partial x}\left(x^{\frac{m+1}{2}} f\right) \\
& =\left(\frac{2}{m+1} \frac{\nu u_{e}}{x}\right)^{1 / 2} \times \frac{1}{2}\left[(m+1) f+2 \bar{x} f_{\bar{x}}+(m-1) \eta f_{\eta}\right]
\end{aligned}
$$$$
u=\frac{\partial \psi}{\partial y}=u_{e} f_{\eta}=u_{\infty}(\bar{x})^{m} f_{\eta}
$$$$
v=-\frac{\partial \psi}{\partial x}=-\left(\frac{2}{m+1} \frac{\nu u_{e}}{x}\right)^{1 / 2} \times \frac{1}{2}\left[(m+1) f+2 \bar{x} f_{\bar{x}}+(m-1) \eta f_{\eta}\right]
$$$$
u_{x}=\frac{u_{e}}{x}\left[m f_{\eta}+\bar{x} f_{\eta \bar{x}}+\left(\frac{m-1}{2}\right) \eta f_{\eta \eta}\right]
$$$$
u_{y}=\left(\frac{m+1}{2} \frac{u_{e}}{x \nu}\right)^{1 / 2} u_{e} f_{\eta \eta}
$$$$
u_{y y}=\left(\frac{m+1}{2}\right) \frac{u_{e}^{2}}{x \nu} f_{\eta \eta \eta}
$$$$
\left(u_{e}\right)_{x}=\frac{m u_{e}}{x}
$$$$
u-u_{e}=u_{e}\left(f_{\eta}-1\right), \quad f_{\eta}=F, \quad f_{\eta \eta}=F_{\eta}, \quad f_{\eta \eta \eta}=F_{\eta \eta}
$$$$
T=T_{\infty}+b_{1}(\bar{x})^{n} G(\bar{x}, \eta)
$$$$
T_{x}=\frac{(\bar{x})^{n}}{x} b_{1}\left[\bar{x} G_{\bar{x}}+\left(\frac{m-1}{2}\right) \eta G_{\eta}+n G\right]
$$$$
T_{y}=b_{1}(\bar{x})^{n}\left(\frac{m+1}{2} \frac{u_{e}}{x \nu}\right)^{1 / 2} G_{\eta}
$$$$
T_{y y}=b_{1}(\bar{x})^{n}\left(\frac{m+1}{2} \frac{u_{e}}{x \nu}\right) G_{\eta \eta}
$$ 


$$
\begin{aligned}
& C=C_{\infty}+b_{2}(\bar{x})^{n} H(\bar{x}, \eta) \\
& C_{x}=\frac{(\bar{x})^{n}}{x} b_{2}\left[\bar{x} H_{\bar{x}}+\left(\frac{m-1}{2}\right) \eta H_{\eta}+n H\right]
\end{aligned}
$$

$$
\begin{aligned}
& C_{y}=b_{2}(\bar{x})^{n}\left(\frac{m+1}{2} \frac{u_{e}}{x \nu}\right)^{1 / 2} H_{\eta} \\
& T_{y y}=b_{2}(\bar{x})^{n}\left(\frac{m+1}{2} \frac{u_{e}}{x \nu}\right) H_{\eta \eta}
\end{aligned}
$$

